\title{
Phonon Spectroscopy at Atomic Resolution
}

\author{
F. S. Hage, ${ }^{1, *}$ D. M. Kepaptsoglou, ${ }^{1,2}$ Q. M. Ramasse, ${ }^{1,3, \dagger}$ and L. J. Allen ${ }^{4,5, \$}$ \\ ${ }^{1}$ SuperSTEM Laboratory, SciTech Daresbury Campus, Daresbury WA4 4AD, United Kingdom \\ ${ }^{2}$ York JEOL Nanocentre and Department of Physics, University of York, Heslington, York YO10 5BR, United Kingdom \\ ${ }^{3}$ School of Physics and School of Chemical and Process Engineering, University of Leeds, Leeds LS2 9JT, United Kingdom \\ ${ }^{4}$ School of Physics, University of Melbourne, Parkville, Victoria 3010, Australia \\ ${ }^{5}$ Ernst Ruska-Centre for Microscopy and Spectroscopy with Electrons, Forschungszentrum Jülich, 52425 Jülich, Germany
}

(Received 26 September 2018; published 10 January 2019)

\begin{abstract}
Advances in source monochromation in transmission electron microscopy have opened up new possibilities for investigations of condensed matter using the phonon-loss sector of the energy-loss spectrum. Here, we explore the spatial variations of the spectrum as an atomic-sized probe is scanned across a thin flake of hexagonal boron nitride. We demonstrate that phonon spectral mapping of atomic structure is possible. These results are consistent with a model for the quantum excitation of phonons and confirm that $Z$-contrast imaging is based on inelastic scattering associated with phonon excitation.
\end{abstract}

DOI: 10.1103/PhysRevLett.122.016103

Improvements in monochromation of electron sources and in spectrometer designs mean that an energy resolution of the order of $10 \mathrm{meV}$ or better is now achievable in experimental electron energy-loss spectroscopy (EELS) [1-3]. This allows the details of energy-loss spectra to be observed down to $\mathrm{THz}$ frequencies, meaning that vibrational spectra associated with phonon excitations are accessible.

Opening up the phonon-loss sector is of importance in physics and materials science since this will allow detailed studies of bonding arrangements, thermal and optical transport, as well as the detection of hydrogen and other light elements directly in the electron microscope. The atomic-scale focused coherent probe in scanning transmission electron microscopy (STEM) allows the vibrational information to be obtained from nanometer-sized regions, making it possible to detect localized vibrational excitations and states. There have been several prospective explorations of these possibilities [4-7] and initial experimental applications have included the measurement of vibrational modes of an ionic liquid [2], the detection of water and its derivatives on individual nanoparticles [8], and the mapping of vibrational surface and bulk modes in a single $\mathrm{MgO}$ nanocube [9]. Further methodological developments have enabled local temperature measurements based on energy gain and loss spectroscopy [10], while momentum-resolved vibrational-loss spectroscopy has recently demonstrated the ability to map both acoustic and optical phonons across the first Brillouin zone of a crystal using a nanometer-sized electron probe $[11,12]$.

Several authors have considered whether phonon scattering could give rise to higher resolution signals $[6,7$, 13-16], taking full advantage of the atomic-sized probe in STEM. This issue has been discussed vigorously since energy losses in the phonon sector are small (of the order of 1-500 meV for single phonon excitation), implying significant delocalization. However, as discussed in Ref. [16], atomically resolved vibrational EELS should be possible since atomic resolution high-angle annular dark-field (HAADF) imaging, also known as Z-contrast imaging, detects electrons which have been scattered to large angles after the excitation of phonons, as we will confirm in this Letter.

Dwyer et al. [17] demonstrated, using a specimen of hexagonal boron nitride, that a focused beam of highenergy electrons can map the vibrational modes of a material with a spatial resolution better than two nanometers. Hexagonal boron nitride is a polar dielectric that gives rise to both localized (impact) and delocalized (dipole) electronvibrational scattering. The dipole scattering gives rise to a background in the images. Dwyer showed that an annular collection geometry should significantly reduce the dipole background, thereby reducing the electron dose required to observe atomic-scale contrast in phonon images [18].

In this Letter, we show that a resolution an order of magnitude better than in Ref. [17] is possible, also using a specimen of hexagonal boron nitride. We demonstrate that electrons in the phonon sector of the energy-loss spectrum provide atomic resolution structure maps of the specimen when the detector is moved off the optical axis and outside the bright-field disk to angular ranges used for typical $Z$-contrast imaging detection geometries. The variation in such spectra with the probe positioned both on and off atomic columns is investigated and the underlying physics is discussed. The structure maps can only be understood using the quantum excitation of phonons (QEP) model [19] and not in the context of the widely used frozen phonon model $[20,21]$. The frozen phonon model is often used to 
model $Z$-contrast imaging assuming elastic scattering from different atomic configurations but the results here show unambiguously that it is inelastically scattered electrons, involved in phonon excitation, which form such images.

Energy-loss spectra were acquired at an acceleration voltage of $60 \mathrm{kV}$ and a probe convergence semiangle of $31.5 \mathrm{mrad}$, resulting in a $1 \AA$ probe size, using a Nion UltraSTEM 100MC scanning transmission electron microscope, equipped with a Gatan Enfinium ERS EEL spectrometer. The different detector positions $A-C$ indicated in Fig. 1(a) were obtained by using a combination of microscope postspecimen deflectors to shift the diffraction pattern with respect to the spectrometer entrance aperture (and HAADF detector). The semiangle of the detectors (i.e., the spectrometer entrance aperture) is $22 \mathrm{mrad}$ in each case. More experimental parameters are given in [22].

Figure 1(b) shows low-loss spectra averaged over the detector for three of the geometries indicated in Fig. 1(a) and normalized by the total acquisition time. The signal is clearly much larger for the detector at the bright-field (BF) position than at the off-axis positions ( $A$ or $B$ ), especially for the zero-loss peak (ZLP). Its full width at half maximum (a commonly used measure of the achieved energy resolution) is slightly increased at positions $A$ and $B(52 \mathrm{meV}$, compared to $28 \mathrm{meV}$ at the $\mathrm{BF}$ position) but this is primarily due to a combination of instrumental factors, as discussed in Ref. [11].

More importantly, phonon-loss peaks are clearly observable between 50-200 meV: both longitudinal $(L)$ and transverse $(T)$ acoustic $(A)$ and optical $(O)$ phonon branches and surface phonon polaritons (PPs) [11,12]. PPs appear at energy losses between the $T O$ and $L O$ bulk modes $[11,12,17,23]$. The PP contribution is significant for small wave vectors $\mathbf{q}$ when the detector is at the $\mathrm{BF}$ position, where by comparison the contribution of the acoustic modes is expected to be small. The energy resolution chosen here, to maximize the signal-to-noise ratio, leads to a single optical " $L O-T O$-PP" loss peak when the detector is at the BF position. Similarly, at the $A$ or $B$ position, we observe acoustic " $L A-T A$ " and optical "LO-TO" loss peaks.

For detector position $A$ in Fig. 1(a), corresponding to a $77 \mathrm{mrad}$ displacement and located on one of the main Kikuchi bands, the zero-loss peak in Fig. 1(b) is substantially reduced and the phonon sector of the spectrum is better exposed, wherein the acoustical phonon contributions between 50 and $100 \mathrm{meV}$ are evident. Moving the detector to position $B$, also displaced by $77 \mathrm{mrad}$ but located between the major Kikuchi bands, further reduces the contribution due to elastic scattering, while some subtle changes in the phonon fine structure can be observed. If instead of displacing the detector an annular entrance aperture of similar angular range were used [18] any azimuthal variation in spectral features and intensities would effectively be averaged out and the resulting
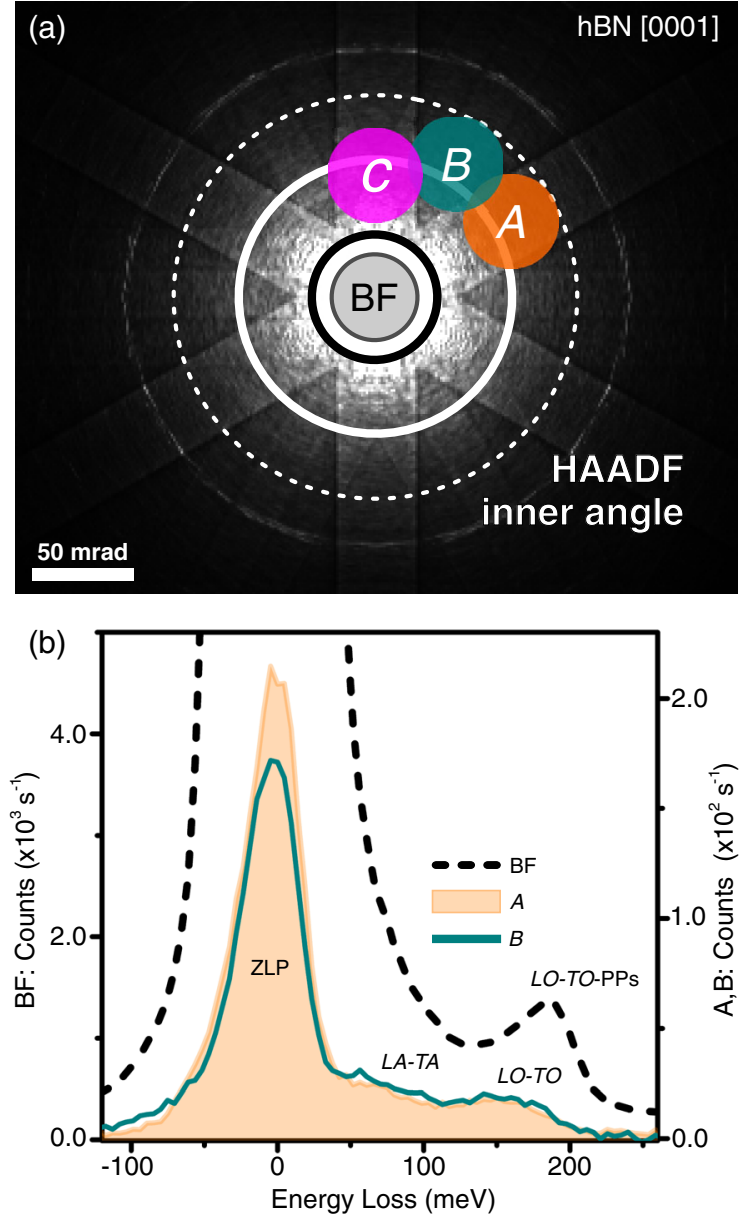

FIG. 1. (a) Schematic showing four detector positions labeled $\mathrm{BF}, A, B$, and $C$ relative to a simulated convergent-beam electron diffraction pattern for hexagonal $\mathrm{BN}$ in [0001] orientation and of $15 \mathrm{~nm}$ thickness, as used in the experiments. The solid black circle shows the extent of the bright-field disk $(31.5 \mathrm{mrad}$ semiangle), while the inner angle of a typical HAADF detector is indicated by a solid white line at $70 \mathrm{mrad}$ semiangle. For practical reasons, the optical conditions used for these experiments resulted in a slightly wider $(100 \mathrm{mrad})$ HAADF inner angle, indicated by a dotted white circle. The detector positions are achieved by deflecting the beam post specimen. (b) Energyloss spectra averaged over the detector at three of the positions indicated in (a), using otherwise identical acquisition parameters, normalized by total acquisition time and scaled as indicated.

spectrum would be expected to resemble an average of spectra $A$ and $B$ in Fig. 1(b).

In Fig. 2(a) we explore the sensitivity of the vibrationalloss spectrum to probe position for the aperture at position $C$ (almost equivalent to position $A$ but displaced by $61 \mathrm{mrad}$ ), as the probe is scanned across an area of the hexagonal boron nitride flake oriented in a [0001] zone axis. In this orientation of the $A A^{\prime}$ 'stacked hexagonal $\mathrm{BN}$ flake, atomic columns consisting of alternating $\mathrm{B}$ and $\mathrm{N}$ atoms through the thickness of the sample are arranged in a honeycomb pattern [24,25]. The flake thickness was 


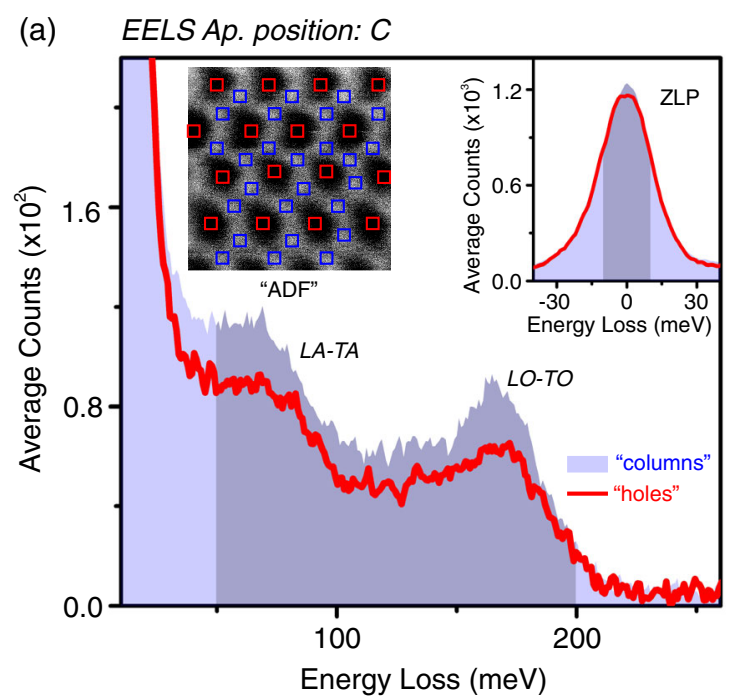

(b) EELS Ap. position: BF

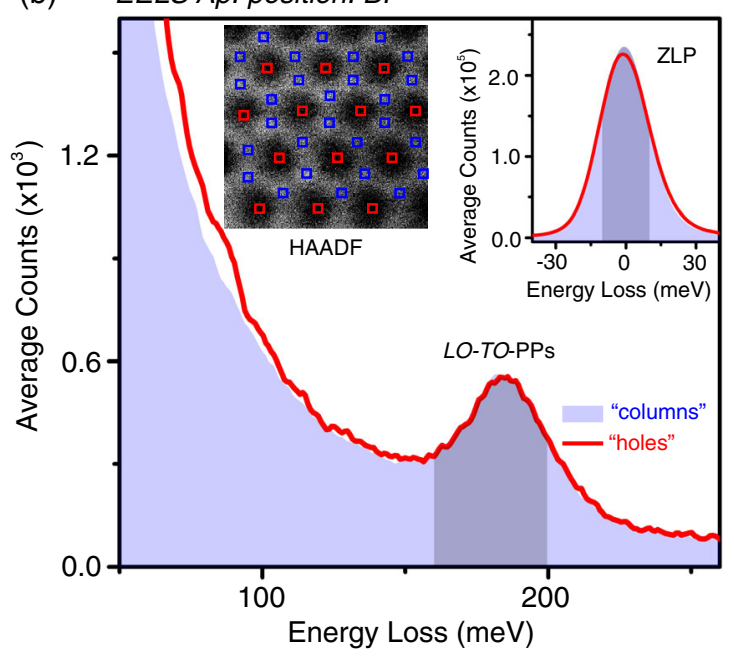

FIG. 2. (a) Low-loss spectra for the detector in position $C$ averaged over probe positions on the atomic columns, labeled "columns" (blue squares on the inset on left) and in the holes between the columns (red squares), labeled "holes." The whole zero-loss peak is shown in the inset on the right. (b) The same as (a) except that the detector is now in the BF position. Energy-loss ranges used for mapping are indicated in both (a) and (b) by shaded areas.

estimated to be $15 \pm 3 \mathrm{~nm}$, corresponding to between 40 and $50 \mathrm{BN}$ layers. The result labeled columns is obtained by averaging spectra recorded when the probe is on the columns (blue squares in the inset on the left-hand side) and that labeled holes when the probe is between the columns (red squares). There is a clear dependence of the signal on probe position, with the intensity of the phonon sector of the spectrum increasing noticeably when the probe is on the columns as the EELS aperture is positioned off axis in position $C$.

When the EELS aperture is on axis in the BF position, Fig. 2(b), the LA-TA peak seen in Fig. 2(b) is no longer evident. This is consistent with calculations that suggest the $L O$-TO-PPs peaks at approximately $170 \mathrm{meV}$ are the dominant contribution to spectra recorded in these experimental conditions $[11,12]$. Furthermore, the sensitivity to probe position of the spectrum in the $L O-T O$-PPs region is now strongly damped. A further feature in Fig. 2(b) is an increase in signal within the tail of the zero-loss peak, below about $100 \mathrm{meV}$, when the probe is in the holes between the columns compared to when it is positioned on the columns. We will elaborate on this in the discussion of Fig. 4.

In view of the clear dependence of the spectrum on probe position observed in Fig. 2(a), it makes sense to form EELS maps by integrating the signal over the energy-loss sectors indicated in the spectra. Shown in Fig. 3(a), where the detector is in position $C$, are the maps from the quasielastic zero-loss peak, $-10 \rightarrow 10 \mathrm{meV}$, and the phonon sector, with the range $50 \rightarrow 200 \mathrm{meV}$ encompassing all the phonon modes expected to dominate the spectrum intensity in this geometry [12]. These can be compared with the signal simultaneously recorded by the HAADF detector, which due to the diffraction pattern shift is not symmetrically placed, hence the nomenclature "ADF." Note that separate maps of the optical or of the acoustic peaks alone using narrower integration windows show the same overall contrast as the $50 \rightarrow 200 \mathrm{meV}$ map, but with poorer signal to noise (see the additional data provided in [22]). The contrast of the phonon sector map mirrors that of the ADF image, significantly different from that of the ZLP mapsee also [22], and is consistent with the map in Fig. 3(a) being dominated by inelastic phonon scattering.

Similar results are shown in Fig. 3(b) for the detector in position $\mathrm{BF}$, but the integration of the phonon sector covers a different range $160 \rightarrow 200 \mathrm{meV}$, that which includes the optical phonon energy losses known to dominate the spectrum in the on-axis geometry [12]. This map shows the lack of contrast consistent with the discussion of Fig. 2(b). Clearly, if phonon excitation events with suitably large momentum transfers are used to form the image then atomic resolution mapping is possible, something which is self-evident if one accepts that a conventional HAADF detector is effectively integrating over the phonon spectrum for a suitably large range of $\mathbf{q}$ values to yield an atomic resolution image. Here, we have shown that this is also possible for a range of somewhat smaller $\mathbf{q}$ values for the detector position $C$.

The interpretation of the experimental results is further validated in Fig. 4(a) by simulations for a $15 \mathrm{~nm}$ thick slab of $A A^{\prime}$ '-stacked hexagonal boron nitride, carried out using the QEP model [19], when the convergent-beam diffraction (CBED) pattern expected on the detector is effectively shifted so that position $C$ coincides with the detector in the forward direction (top right panel)-i.e., by a magnitude of $61 \mathrm{mrad}$ in the direction shown. The left panel shows the spectrum map for phonons scattered into the $22 \mathrm{mrad}$ 


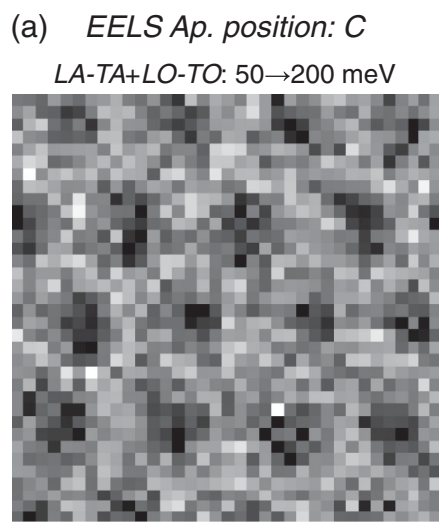

(b) EELS Ap. position: BF LO-TO-PPs: $160 \rightarrow 200 \mathrm{meV}$

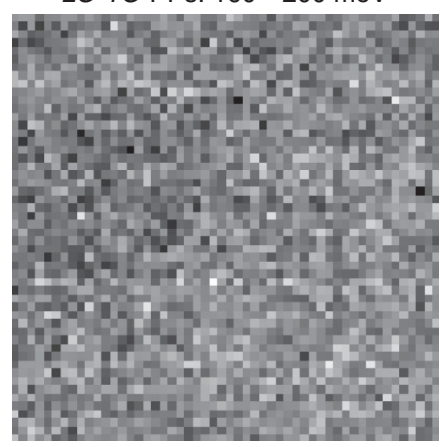

ZLP: $-10 \rightarrow 10 \mathrm{meV}$

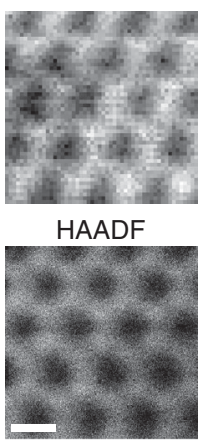

FIG. 3. (a) EELS intensity maps formed by signal integration over the energy windows indicated in Fig. 2(a) (detector at position $C$ ) and (b) for the energy windows indicated in Fig. 2(b) (detector in BF position). Scale bars: $2 \AA$.

detector. These results are calculated using an Einstein model - to a very good approximation, the same as the integrated spectrum in a correlated model [26]. Last, we have the signal into the HAADF detector, again denoted ADF.

Figure 4(b) is the same as Fig. 4(a) but for no deflection of the diffraction pattern. Note that in this case the contrast of the spectrum map for scattering into the 22 mrad detector is reduced by a factor of $\approx 3$, defined in terms of the maximum and minimum intensity by $\left(I_{\max }-I_{\min }\right) /$ $\left(I_{\max }+I_{\min }\right)$. Each of Figs. 4(a) and 4(b) is plotted on its own gray scale for visibility, so that the contrast in Fig. 4(b) is "stretched" relative to that in Fig. 4(a) and artificially appears stronger than it is in absolute terms. The simulations in Fig. 4 were done taking into account the high resolution (impact) scattering. The slowly varying background due to the delocalized dipole scattering $[18,27]$, important for small momentum transfers $\mathbf{q}$, further diminishes contrast, consistent with the experimental results shown in the left panel of Fig. 3(b).

Simulations exploring the angular dependence of the contributions to the contrast of the spectrum map in Fig. 4(b) are shown in [22]. The contrast reversal for $0 \lesssim 15 \mathrm{mrad}$ is consistent with the observation, made when discussing Fig. 2(b), that there is more signal for energy (a) EELS Ap. position: $C$

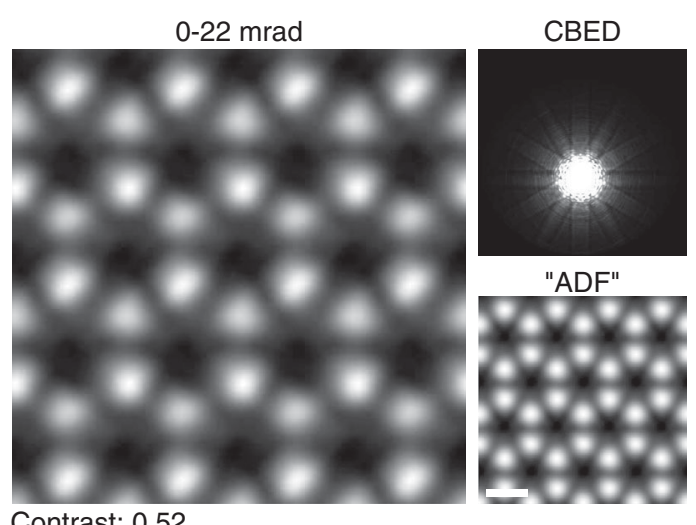

Contrast: 0.52

(b) EELS Ap. position: BF

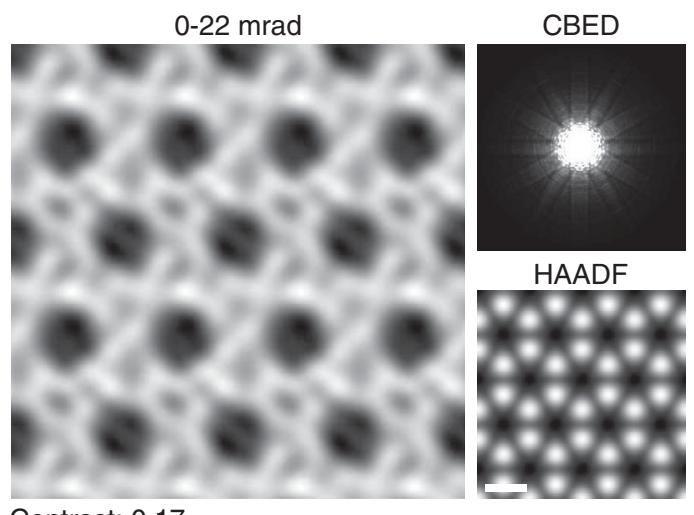

Contrast: 0.17

FIG. 4. (a) Simulations of the CBED pattern tilted to effectively put the detector, in the forward direction, in position $C$; of the spectrum map into the $22 \mathrm{mrad}$ detector; and of the image into the ADF detector. (b) The same as (a) but the detector is in the BF position. The contrast is defined in terms of the maximum and minimum intensity by $\left(I_{\max }-I_{\min }\right) /$ $\left(I_{\max }+I_{\min }\right)$. Scale bars: $2 \AA$.

losses $\lesssim 100 \mathrm{meV}$ when the probe is in the holes between the columns; smaller momentum transfers are correlated with smaller energy losses [26]. The contributions from the (larger area) annular region from approximately 15 to $22 \mathrm{mrad}$ (and which correlates with larger energy losses), where there is more signal on the columns, dominates the contrast to give the result shown in the left panel of Fig. 4(b). The slight minima on atomic sites in Fig. 4(b) are attributable to more scattering to larger $\mathbf{q}$ when the probe couples to a column.

In conclusion, we have shown that there are atomicscale variations in the vibrational-loss spectrum from a thin flake of hexagonal boron nitride as an atomic-sized probe is scanned across the specimen. In particular, spectral mapping of atomic structure using the phonon sector of the energy-loss spectrum is demonstrated for an off-axis collection geometry, a setup which also suppresses the contribution due to dipole scattering. Possible future 
applications of this technique include mapping of localized, defect-induced, finite $\mathbf{q}$ vibrational modes as well as similar local modifications of the vibrational response of materials. Additionally, these results support the quantum excitation of phonons model and confirm that the standard technique of $Z$-contrast imaging is based on the inelastic scattering associated with phonon excitations, not consistent with the frozen phonon model which assumes elastic scattering from different configurations of the atoms in the specimen.

This research was supported under the Discovery Projects funding scheme (Project No. DP140102538) of the Australian Research Council and by the Alexander von Humboldt Foundation. L. J. A. thanks Dr. Hamish Brown and Dr. Scott Findlay for helpful discussions. SuperSTEM is the U.K. Engineering and Physical Sciences Research Council (EPSRC) National Research Facility for Advanced Electron Microscopy.

*fshage@superstem.org

qmramasse@superstem.org

*lja@unimelb.edu.au

[1] O. L. Krivanek, T. C. Lovejoy, N. Dellby, T. Aoki, R. W. Carpenter, P. Rez, E. Soignard, J. Zhu, P. E. Batson, M. J. Lagos, R. F. Egerton, and P. A. Crozier, Nature (London) 514, 209 (2014).

[2] T. Miyata, M. Fukuyama, A. Hibara, E. Okunishi, M. Mukai, and T. Mizoguchi, Microscopy 63, 377 (2014).

[3] T. C. Lovejoy, G. C. Corbin, N. Dellby, M. V. Hoffman, and O. L. Krivanek, Microsc. Microanal. 24, 446 (2018).

[4] O. Krivanek, N. Dellby, T. Lovejoy, N. Bacon, G. Corbin, P. Hrncirik, Z. S. Szilagyi, T. Aoki, R. W. Carpenter, and P. A. Crozier et al., Microsc. Microanal. 20, 66 (2014).

[5] P. A. Crozier, J. Zhu, T. Aoki, P. Rez, W. J. Bowman, R. W. Carpenter, O. L. Krivanek, N. Dellby, T. C. Lovejoy, and R. F. Egerton, Microsc. Microanal. 20, 72 (2014).

[6] R. F. Egerton, Microsc. Microanal. 20, 658 (2014).

[7] R. Egoavil, N. Gauquelin, G. T. Martinez, S. Van Aert, G. Van Tendeloo, and J. Verbeeck, Ultramicroscopy 147, 1 (2014).

[8] P. A. Crozier, T. Aoki, and Q. Liu, Ultramicroscopy 169, 30 (2016).

[9] M. J. Lagos, A. Trügler, U. Hohenester, and P. E. Batson, Nature (London) 543, 529 (2017).
[10] J. C. Idrobo, A. R. Lupini, T. Feng, R. R. Unocic, F. S. Walden, D. S. Gardiner, T. C. Lovejoy, N. Dellby, S. T. Pantelides, and O. L. Krivanek, Phys. Rev. Lett. 120, 095901 (2018).

[11] F. S. Hage, R. J. Nicholls, J. R. Yates, D. G. McCulloch, T. C. Lovejoy, N. Dellby, O. L. Krivanek, K. Refson, and Q. M. Ramasse, Sci. Adv. 4, eaar7495 (2018).

[12] R. J. Nicholls, F. S. Hage, D. G. McCulloch, Q. M. Ramasse, K. Refson, and J. R. Yates, arXiv:1804.10475.

[13] P. Rez, Ultramicroscopy 52, 260 (1993).

[14] C. Dwyer, Phys. Rev. B 89, 054103 (2014).

[15] P. Rez, Microsc. Microanal. 20, 671 (2014).

[16] N. R. Lugg, B. D. Forbes, S. D. Findlay, and L. J. Allen, Phys. Rev. B 91, 144108 (2015).

[17] C. Dwyer, T. Aoki, P. Rez, S. L. Y. Chang, T. C. Lovejoy, and O. L. Krivanek, Phys. Rev. Lett. 117, 256101 (2016).

[18] C. Dwyer, Phys. Rev. B 96, 224102 (2017).

[19] B. D. Forbes, A. V. Martin, S. D. Findlay, A. J. D’ Alfonso, and L. J. Allen, Phys. Rev. B 82, 104103 (2010).

[20] R. F. Loane, P. Xu, and J. Silcox, Acta Crystallogr. Sect. A 47, 267 (1991).

[21] E. J. Kirkland, Advanced Computing in Electron Microscopy (Springer Science \& Business Media, New York, 2010).

[22] See Supplemental Material at http://link.aps.org/ supplemental/10.1103/PhysRevLett.122.016103 for more experimental parameters; Fig. S1, experimental off-axis spectrum maps of the LA-TA and LO-TO phonon-loss peaks, and Fig. S2, annular contributions to Fig. 4(b) over the ranges $0-15 \mathrm{mrad}$ and $15-22 \mathrm{mrad}$.

[23] A. A. Govyadinov, A. Konečná, A. Chuvilin, S. Vélez, I. Dolado, A. Y. Nikitin, S. Lopatin, F. Casanova, L. E. Hueso, J. Aizpurua, and R. Hillenbrand, Nat. Commun. 8, 95 (2017).

[24] A. Shmeliov, J. S. Kim, K. B. Borisenko, P. Wang, E. Okunishi, M. Shannon, A. I. Kirkland, P. D. Nellist, and V. Nicolosi, Nanoscale 5, 2290 (2013).

[25] G. Constantinescu, A. Kuc, and T. Heine, Phys. Rev. Lett. 111, 036104 (2013).

[26] B. D. Forbes and L. J. Allen, Phys. Rev. B 94, 014110 (2016).

[27] L. J. Allen, H. G. Brown, S. D. Findlay, and B. D. Forbes, Microscopy 67, i24 (2018). 